\title{
Calculation of Admittance Capacity of Wind Farms under Harmonic Constraints
}

\author{
GUO Min ${ }^{\mathrm{a},}{ }^{*}$, JIN Qingren ${ }^{\mathrm{b}}$, and CHEN Weidong ${ }^{\mathrm{c}}$ \\ Electric Power Research Institute of Guangxi Power Grid Co., Ltd., Nanning, Guangxi of China
}

\begin{abstract}
The harmonic voltage at the wind farm interconnection point does not exceed the harmonic distortion limits of all voltage levels stipulated by the national standard. The allowable value of the harmonic current injected into the power system shall be allocated in proportion to the total capacity and installed capacity of the harmonic source equipment. However, judging from the status quo of the implementation of harmonic national standard, there is still much controversy over this standard. One of the focal points of controversy is determining the limits of whether different types of harmonic sources should be treated differently. Based on the theoretical research, this paper selects the typical wind farm interconnection points and calculates the admittance capacity limits under different harmonic constraints of the wind farm based on the historical data and the level of harmonic planning, and analyzes the rationality of the calculation results.
\end{abstract}

\section{Introduce}

The operating characteristics of wind power are different from conventional power supplies. The randomness and intermittency of wind energy determine that the output characteristics of wind turbines are also fluctuating and intermittent ${ }^{[1-4]}$. The asynchronous generator, as a power generating mechanism, injects some harmonics into the system while sending active power, and its harmonic content changes according to the capacity of the wind farm. When the capacity of the wind farm is small, the impact of these characteristics on the power system is not significant, but as the proportion of wind farm capacity in the system increases, the impact of the wind farm on the system will become more and more obvious ${ }^{[5-9]}$. For this reason, this article calculates the admittance capacity of the wind farm to build based on harmonic content requirements of interconnection points.

\section{Calculation steps}

Under harmonic constraints, the calculation process of wind farm grid-connected and grid-connected capacity of photovoltaic power plants can be summarized as follows:

1) Query the total available power parameter value of the network at the network connection point, and obtain the $\mathrm{h}$ ( $\mathrm{h}$ is 2 to 19 ) harmonic emission limit values based on the historical data;

2) According to the h-th harmonic planning level, the recommended value of the superposition coefficient and the recommended coefficient at the same time, calculate the admittance of wind farm grid-connected and photovoltaic power station under the $\mathrm{h}$-th harmonic constraint Capacity limit;

3) Take the minimum value from the second harmonic to the 19th harmonic constraint as the maximum admittance capacity for wind power or photovoltaic power station access at the grid connection point.

\section{Wind farm network introduction}

The wind farm intends to access $110 \mathrm{kV}$ busbar of a $220 \mathrm{kV}$ substation. There are two main transformers, high voltage side with double bus wiring and with 6 feeders. The mid-voltage side $110 \mathrm{kV}$ system uses 12 bus lines with double busbar, and the low voltage side of the substation is wired with single busbar. The configured $35 \mathrm{kV}$ reactive power compensation capacitor adopts outdoor parallel capacitor.

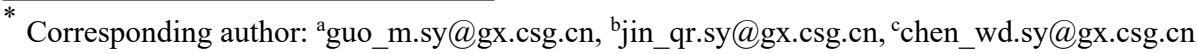




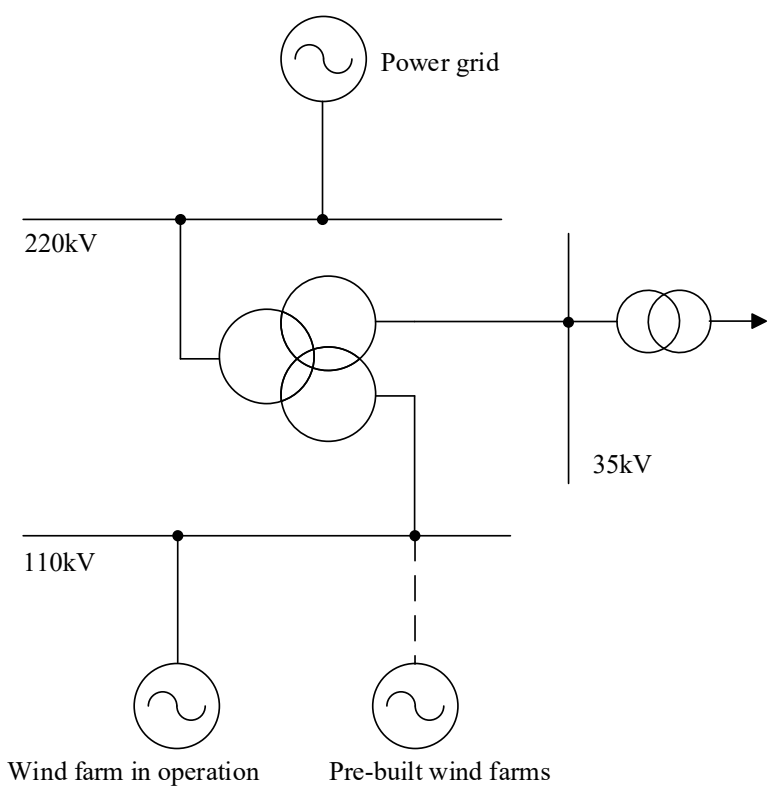

Fig. 1. The main electrical connection of the substation

\section{Parameter acquisition process}

Take the 5th harmonic as an example to illustrate the method of setting the 5th harmonic voltage emission limit EU5. Harmonic voltage monitoring has been carried out on the three wind power integration points (line 197, line 191 and line 154) which have been operated. The statistical results of the fifth harmonic voltage content are as follows:

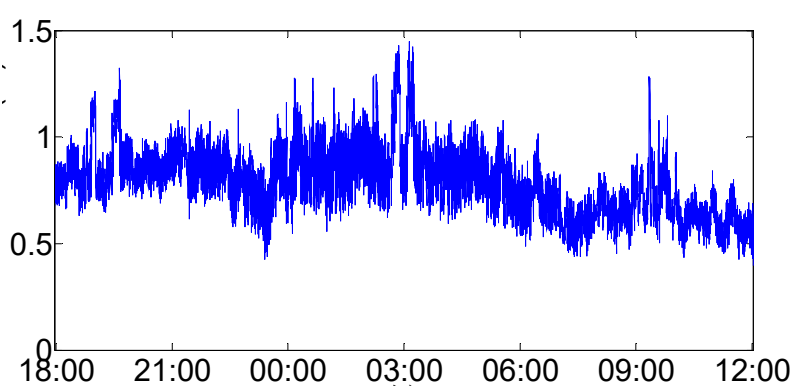

Fig. 2. A-phase 5th harmonic voltage ratio of line 197

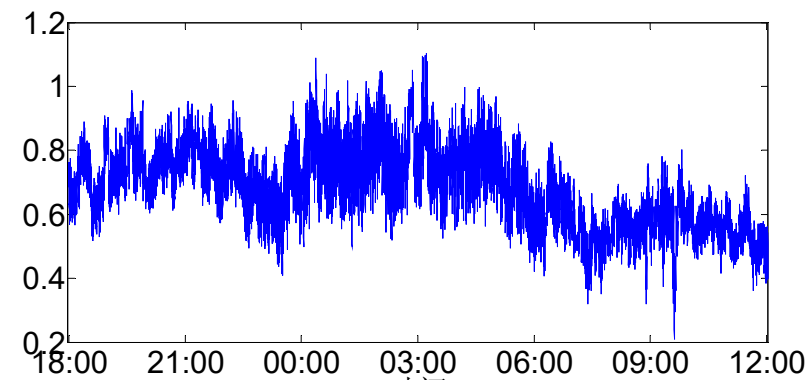

Fig. 3. B-phase 5th harmonic voltage ratio of line 197

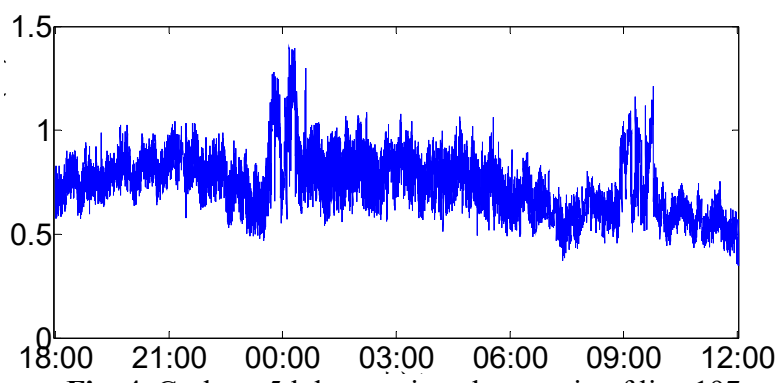

Fig. 4. C-phase 5th harmonic voltage ratio of line 197

The 5th harmonic voltage ratio of line 197 is shown in Figs. 2-4. The average values of the 5th harmonic voltage of A, B and C phases were $0.7908 \%, 0.6842 \%$ and $0.7437 \%$ respectively, and the maximum values were $1.4483 \%, 1.1042 \%$ and $1.3978 \%$ respectively. The $95 \%$ probability value were $1.0405 \%, 0.8802 \%$, $0.9661 \%$.

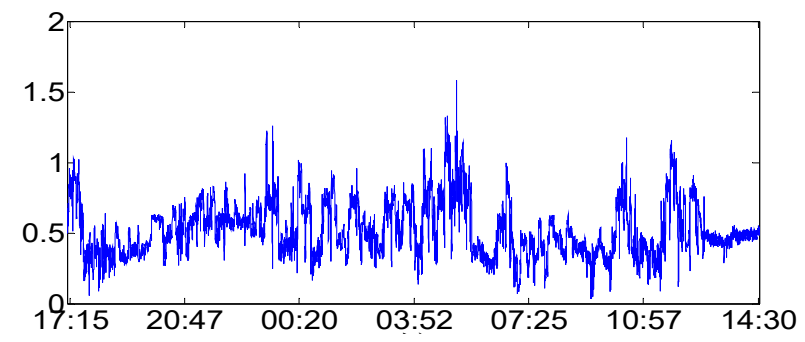

Fig. 5. A-phase 5th harmonic voltage ratio of line 191

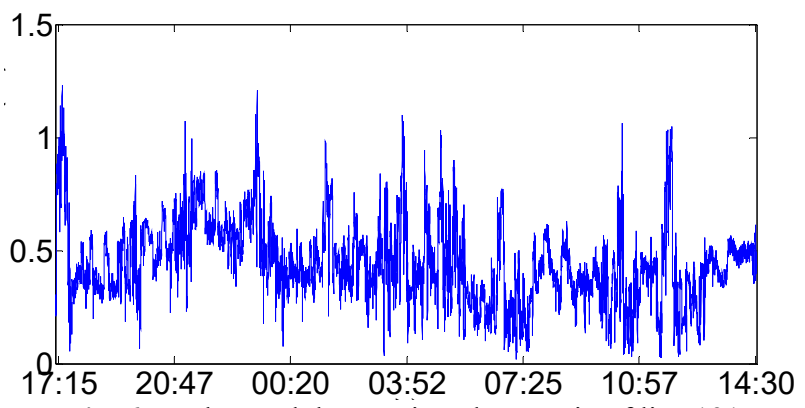

Fig. 6. B-phase 5 th harmonic voltage ratio of line 191

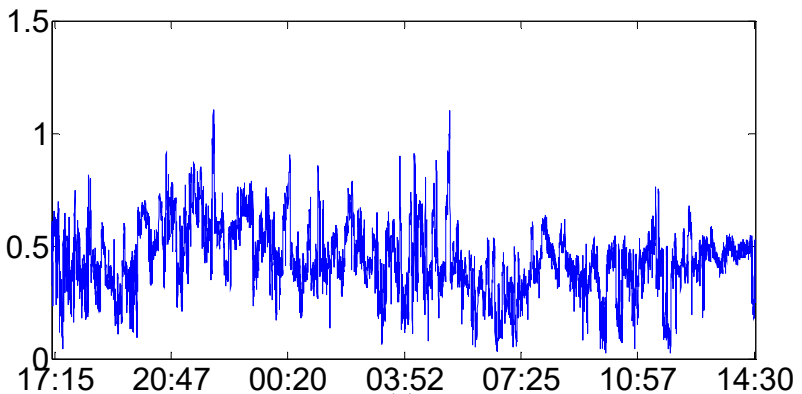

Fig. 7. B-phase 5th harmonic voltage ratio of line 191

The 5th harmonic voltage ratio of line 191 is shown in Figs. 5-7. The average values of the 5th harmonic voltage of A, B and C phases were $0.5239 \%, 0.4510 \%$ and $0.4440 \%$ respectively, and the maximum values were $1.5802 \%, 1.2314 \%$ and $1.1044 \%$ respectively. The $95 \%$ probability values were $0.8632 \%$ and $0.7776 \%$, $0.6955 \%$. 


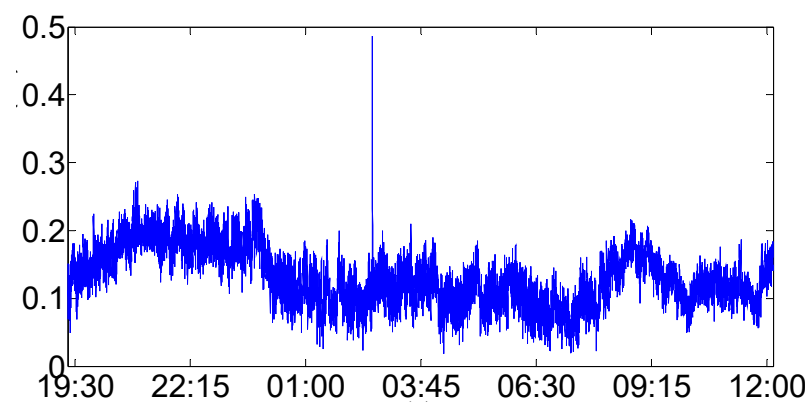

Fig. 8. A-phase 5th harmonic voltage ratio of line 191

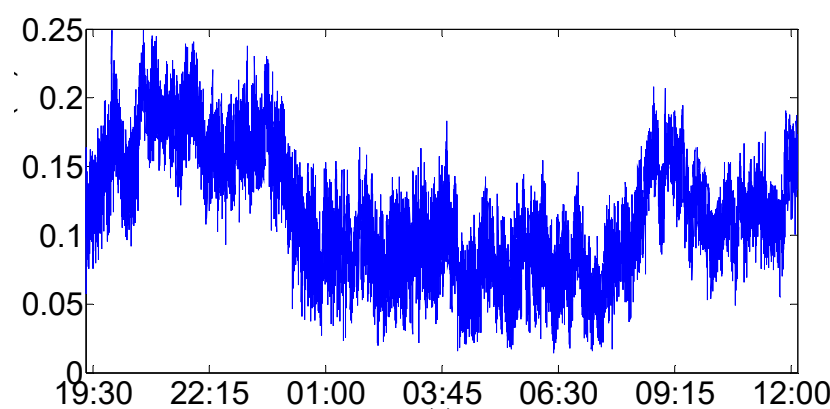

Fig. 9. B-phase 5th harmonic voltage ratio of line 191

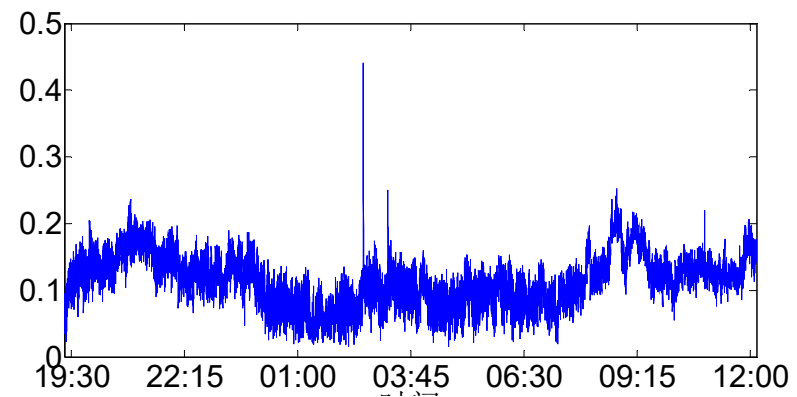

Fig. 10. B-phase 5th harmonic voltage ratio of line 191

The 5 th harmonic voltage ratio of line 154 is shown in Figs. 8-10. The fifth harmonic voltage of A, B and C were $0.1327 \%, 0.1182 \%$ and $0.1152 \%$ respectively, the maximum values were $0.4868 \%, 0.2494 \%$ and $0.4411 \%$, the $95 \%$ probability values were $0.2046 \%, 0.1952 \%$ and $0.1789 \%$, respectively.

Table 1. 5th harmonic voltage maximum ratio in the grid connection point

\begin{tabular}{|c|c|}
\hline The grid connection point & $\begin{array}{c}\text { 5th harmonic voltage } \\
\text { maximum ratio }\end{array}$ \\
\hline Line 197 & $1.4483 \%$ \\
\hline Line 191 & $1.5802 \%$ \\
\hline Line 154 & $0.4868 \%$ \\
\hline
\end{tabular}

The upper limit of EU5 that can be obtained from the above table is $1.5802 \%$. Similarly, the maximum value of the harmonic emission limits for the remaining 2-19 wind farms can be obtained as shown in the following table:

Table 2. The maximum harmonic voltage ratio at the grid connection point

\begin{tabular}{|c|c|}
\hline 2 & 1.7289 \\
\hline 3 & 0.63392 \\
\hline 4 & 0.59711 \\
\hline 5 & 1.5802 \\
\hline 6 & 1.3875 \\
\hline 7 & 1.075 \\
\hline 8 & 0.36957 \\
\hline 9 & 2.2025 \\
\hline 10 & 0.15097 \\
\hline 11 & 0.7915 \\
\hline 12 & 0.1116 \\
\hline 13 & 1.1331 \\
\hline 14 & 0.13758 \\
\hline 15 & 0.55535 \\
\hline 16 & 0.076316 \\
\hline 17 & 0.6443 \\
\hline 18 & 0.11194 \\
\hline 19 & 0.56335 \\
\hline
\end{tabular}

\section{Calculation results}

Now assess the maximum admittance of wind farms under harmonic constraints. Under each harmonic constraint condition, the maximum admittance capacity of the wind farm is shown in Table 7-3, so the maximum admittance capacity of the wind farm at the interconnected site is $41.9337 \mathrm{MVA}$.

Table 3. The maximum admittance capacity of wind farms under various harmonic constraints

\begin{tabular}{|c|c|c|c|c|c|c|}
\hline$h$ & $\begin{array}{c}E_{\mathrm{U} h} \\
(\%)\end{array}$ & $\begin{array}{c}L_{h \mathrm{HV}} \\
(\%)\end{array}$ & $\begin{array}{c}S_{\mathrm{t}} \\
(\mathrm{MVA})\end{array}$ & $F_{\mathrm{HV}}$ & $\alpha$ & $S$ (MVA) \\
\hline 2 & 1.7289 & 1.5 & 360 & 0.8 & 1 & 331.9488 \\
\hline 3 & 0.6339 & 2 & 360 & 0.8 & 1 & 91.2845 \\
\hline 4 & 0.5971 & 1 & 360 & 0.8 & 1 & 171.9677 \\
\hline 5 & 1.5802 & 2 & 360 & 0.8 & 1.4 & 207.0844 \\
\hline 6 & 1.3875 & 0.5 & 360 & 0.8 & 1.4 & 1202.1556 \\
\hline 7 & 1.0750 & 2 & 360 & 0.8 & 1.4 & 120.7598 \\
\hline 8 & 0.3696 & 0.4 & 360 & 0.8 & 1.4 & 257.8006 \\
\hline 9 & 2.2025 & 1 & 360 & 0.8 & 1.4 & 869.9109 \\
\hline 10 & 0.1510 & 0.4 & 360 & 0.8 & 1.4 & 73.6132 \\
\hline 11 & 0.7915 & 0.5 & 360 & 0.8 & 2 & 721.6960 \\
\hline 12 & 0.1116 & 0.2 & 360 & 0.8 & 2 & 89.6728 \\
\hline 13 & 1.1331 & 1.5 & 360 & 0.8 & 2 & 164.3412 \\
\hline 14 & 0.1376 & 0.2 & 360 & 0.8 & 2 & 136.2834 \\
\hline 15 & 0.5554 & 0.3 & 360 & 0.8 & 2 & 986.9236 \\
\hline 16 & 0.0763 & 0.2 & 360 & 0.8 & 2 & 41.9337 \\
\hline 17 & 0.6443 & 1 & 360 & 0.8 & 2 & 119.5553 \\
\hline 18 & 0.1119 & 0.2 & 360 & 0.8 & 2 & 90.2201 \\
\hline 19 & 0.5634 & 1 & 360 & 0.8 & 2 & 91.4006 \\
\hline
\end{tabular}

\section{Summary}

If the harmonic limit of wind power is too loose, the power quality of the power system will drop after the grid is connected, threatening the security of the grid. If the wind power harmonic limit is too strict, it will lead to 
unnecessary waste of funds. This paper selects the typical wind farm interconnection points and calculates the admittance capacity limits under different harmonic constraints of the wind farm based on the historical data and the level of harmonic planning, and analyzes the rationality of the calculation results. It is expected that the characteristics of wind power harmonics will be further studied and a more scientific harmonic limit allocation method will be proposed.

\section{References}

1. Soman, S. S., Zareipour, H., Malik, O., \& Mandal, P. (2010). A review of wind power and wind speed forecasting methods with different time horizons. North American Power Symposium (pp.1-8). IEEE.

2. Gipe, P. (2004). Wind power. Wind Engineering, 28(5), 629-631.

3. Erlich, I., \& Bachmann, U. (2005, June). Grid code requirements concerning connection and operation of wind turbines in Germany. In Power Engineering Society General Meeting, 2005. IEEE (pp. 12531257). IEEE.

4. Yazhou, L. (2003). Studies on wind farm integration into power system [J]. Automation of Electric Power Systems, 8(17), 17.

5. Liang, L., Li, J. L., \& Hui, D. (2011). Optimization configuration for capacity of energy storage system in large-scale wind farm. Gaodianya Jishu/ High Voltage Engineering, 37(4), 930-936.

6. Billinton, R., \& Bai, G. (2004). Generating capacity adequacy associated with wind energy. IEEE transactions on energy conversion, 19(3), 641-646.

7. Liserre, M., Cardenas, R., Molinas, M., \& Rodriguez, J. (2011). Overview of multi-MW wind turbines and wind parks. IEEE Transactions on Industrial Electronics, 58(4), 1081-1095.

8. Mabel, M. C., \& Fernandez, E. (2008). Analysis of wind power generation and prediction using ANN: A case study. Renewable energy, 33(5), 986-992.

9. Ozerdem, B., Ozer, S., \& Tosun, M. (2006). Feasibility study of wind farms: A case study for Izmir, Turkey. Journal of Wind Engineering and Industrial Aerodynamics, 94(10), 725-743. 